\title{
COMPUTER SIMULATION OF A MAGNETIC THIN FILM OF CoCr
}

\author{
E.M.C.M. REUVEKAMP, J.H.J. van OPHEUSDEN and G.J. GERRITSMA \\ University of Twente, Faculty of Applied Physics, P.O. Box 217, 7500 AE Enschede, The Netherlands
}

\begin{abstract}
A discrete micromagnetic model for a thin magnetic layer has been developed to perform computer simulations. The magnetisation in this model is given in terms of a cubic array of interacting microscopic spins. The dynamics of the spins is given by both a time discretisation of the Landau-Lifshitz-Gilbert equations of motion and an energy minimalisation method. In the calculations presented we use a planar $30 \times 30 \times 1$ array for simplicity.
\end{abstract}

\section{Introduction}

The simulations presented, describing micromagnetic phenomena, could be relevant for simulation of magnetic and magneto-optic recording processes on media having large information densities. For example spin configurations as well as macroscopic observables can be determined, linking the simulations to current experimental techniques as Kerr-microscopy, colloid-SEM and VSM. In the model discrete classical magnetic spins, are considered and their dynamical behaviour is studied. The practical limit for our computer system, an Apollo DN-4000 workstation, is of the order of 1000 spins, mainly for reasons of computational speed.

\section{Model}

As a model for micromagnetism a discretised version of the Landau-Lifshitz-Gilbert (LLG) equations of motion is used. The magnetisation in terms of a classical vector field $\boldsymbol{m}(\boldsymbol{r}, t)$ is discretised on a cubic array of classical magnetic spins, with fixed moment $|\boldsymbol{m}|$ but with arbitrary orientation. In the $x$ - and $y$-direction periodical boundary conditions are applied, in the $z$-direction the lattice is finite. The magnetic energy $E\left(\boldsymbol{m}_{1}, \ldots, \boldsymbol{m}_{N}, \boldsymbol{B}_{\mathrm{ext}}\right)$ of the spins contains three essentially different terms: an interaction with the external field $\boldsymbol{B}_{\mathrm{ex} 1}$, with the crystal lattice and a mutual interaction between the spins. The last term has two parts. First there is an exchange energy density of the form [1]

$E_{\text {exchange }}=-\frac{A}{2 a^{2}} \sum_{\text {neighbours }} \hat{\boldsymbol{m}}_{i} \cdot \hat{\boldsymbol{m}}_{j}$,

with $A$ the exchange constant as usually used in continuum describtions, $a$ the cubic lattice spacing and $\hat{\boldsymbol{m}}$ the unit magnetisation vector. Secondly there is a dipole interaction term, of the form

$E_{\text {dipole }}=\frac{\mu_{0}}{4 \pi} \sum_{\text {pairs }}\left[\boldsymbol{m}_{i} \cdot \boldsymbol{m}_{j}-3\left(\boldsymbol{m}_{i} \cdot \hat{\boldsymbol{r}}_{i j}\right)\left(\boldsymbol{m}_{j} \cdot \hat{\boldsymbol{r}}_{i j}\right)\right] / r_{i j}^{3}$,

0304-8853/90/\$03.50 $\odot$ Elsevier Science Publishers B.V

(North-Holland) with $\boldsymbol{r}_{i j}=\boldsymbol{r}_{i}-\boldsymbol{r}_{j}, \hat{\boldsymbol{r}}_{i j}=\boldsymbol{r}_{i j} /\left|\boldsymbol{r}_{i j}\right|$ and $\boldsymbol{r}_{i}$ the Euclidean position of the spins and $\boldsymbol{m}_{i}=a^{3} m_{\mathrm{s}} \hat{\boldsymbol{m}}_{i}$ the discreted magnetic moment, where $m_{\mathrm{s}}$ is the saturation magnetisation. Next there is a phenomenological interaction with the crystal lattice, which we have taken to be a uniaxial anisotropy field

$E_{\text {crystal }}=-\sum_{\text {spins }} K_{i}\left(\hat{\boldsymbol{m}}_{i} \cdot \boldsymbol{a}_{i}\right)^{2}$.

The unit vector $a_{i}$ gives the direction of the easy axis, which can point principally in any direction. Also the interaction strength $K_{i}$ may differ from one spin to another. Both can differ between layers in order to simulate double layer materials, defects and other discontinuities. Finally, the interaction with the external field $\boldsymbol{B}_{\mathrm{ex} 1}$ is given by

$E_{\text {external }}=-m_{\mathrm{s}} \sum_{\text {spins }} \hat{\boldsymbol{m}}_{i} \cdot \boldsymbol{B}_{\text {ext }}$.

The external field can be varied with time in order to investigate the dynamical behaviour under changes in the field, as in hysteresis loops.

The LLG equations include a phenomenological damping term describing the relaxation into the direction of the local magnetic field. The equations of motion for a single spin are of the form

$\frac{\mathrm{d} \boldsymbol{m}}{\mathrm{d} t}=-\gamma \boldsymbol{m} \times \boldsymbol{B}_{\mathrm{loc}}-\frac{\alpha \gamma}{|\boldsymbol{m}|} \boldsymbol{m} \times\left(\boldsymbol{m} \times \boldsymbol{B}_{\mathrm{loc}}\right)$,

where $\gamma$ is the gyromagnetic ratio, $\alpha$ the damping parameter and $\boldsymbol{B}_{\mathrm{loc}}=-\mathrm{d} E / \mathrm{dm}$ the local induction.

\section{Discretisation}

The presented simulations use complete evaluation of the preceding interaction terms. The equations of motion (5) are scaled in time to $\gamma t$ and integrated numerically with first order Euler for finite $\alpha$. This approach makes dynamic studies, of for example microwave absorption, possible in extension to simulation of Schabes, Zhu and Bertram $[2,3]$. In a second type of approach the magnetic energy is minimized, which is 
obtained for $\boldsymbol{m} / / \boldsymbol{B}_{\mathrm{loc}}$ in each iteration step. Either technique is used depending on the type of information desired.

Characteristic values for the parameters of the (CoCr)

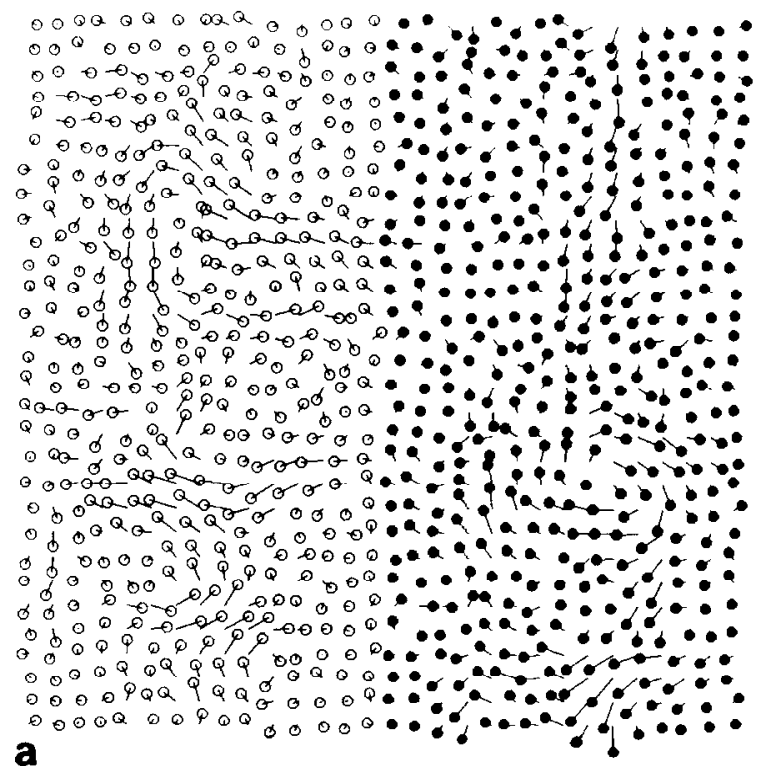

b

$\odot \bullet \bullet \bullet \bullet \bullet \bullet \bullet \bullet \bullet Q \odot \bullet Q^{Q} \bullet \bullet \bullet \bullet \bullet \bullet \bullet Q Q \bullet$

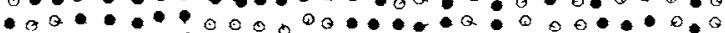

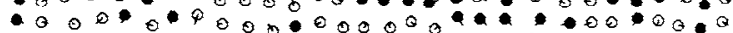

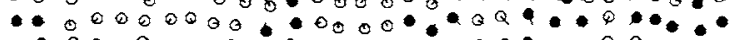

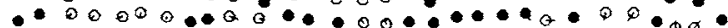

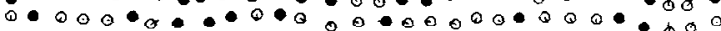

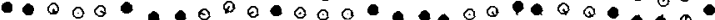
$Q$ $P$ P

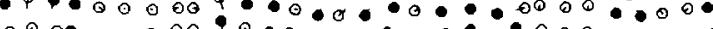

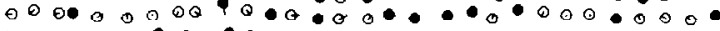

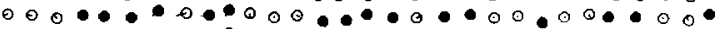
$000000000 \cdots 00 \cdots$ $\bullet \bullet 000 \bullet \bullet \bullet 00000000 \bullet \bullet \bullet \bullet \bullet \bullet \bullet \bullet \bullet \bullet$ $0000 \div 0$

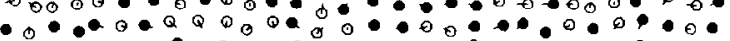

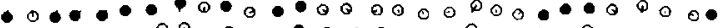

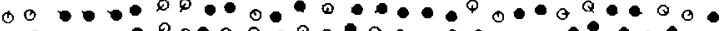

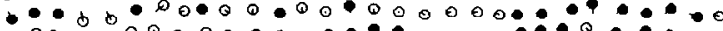

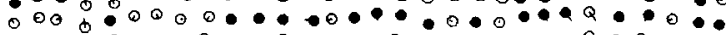

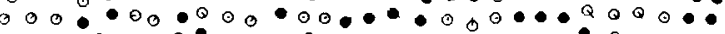

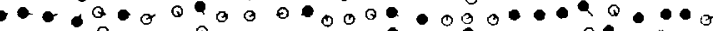

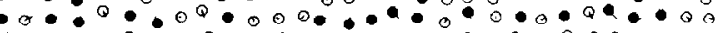

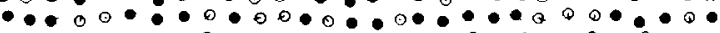

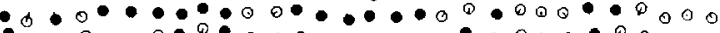

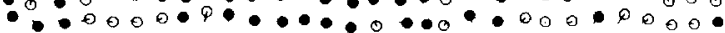

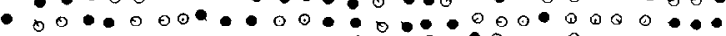

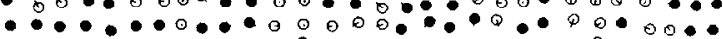

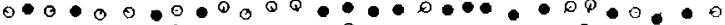

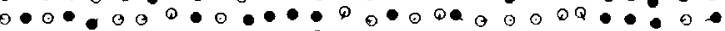

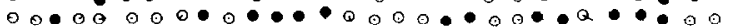

Fig. 1. Relaxed spin configuration in $\boldsymbol{B}_{\mathrm{ext}}=0 \mathrm{~T}$ for parameters as in text, (a) of a stripe-domain using the energy minimalisation method and (b) of a random start-configuration using LLG equations. Open and filled circles have spin up and down, respectively.

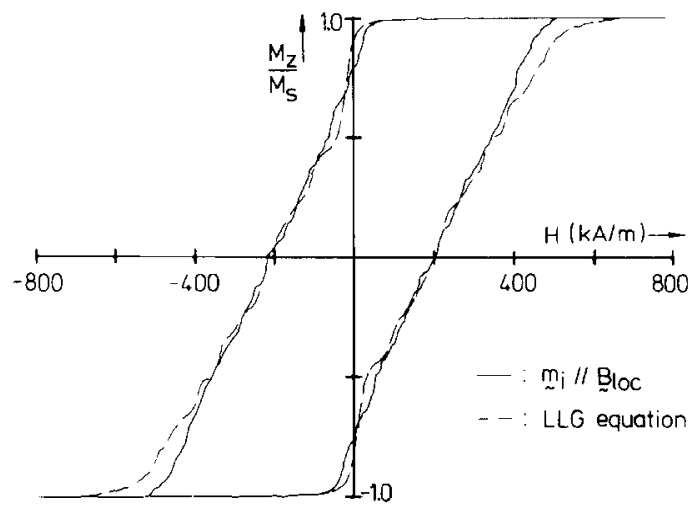

Fig. 2. Hysteresis loops in perpendicular field with parameters as in text. Maximum field $|\boldsymbol{H}|=800 \mathrm{kAm}^{-1}$. Dashed line: energy minimalisation. Solid line: LLG equations.

magnetic film are taken to be: $A \approx 10^{-11} \mathrm{Jm}^{-1}, m_{\mathrm{s}} \approx 4$ $\times 10^{5} \mathrm{Am}^{-1}, K=1.6 \times 10^{5} \mathrm{Jm}^{-3}, a \approx 16 \mathrm{~nm}, d(\gamma t) \approx$ $0.05 \mathrm{~T}^{-1}, \alpha \approx 0.1$.

The easy-axis is taken randomly in a cone, with top-angle $\Delta \theta=6^{\circ}$, around the film-normal. All calculations presented are performed on a $30 \times 30 \times 1$ lattice.

\section{Computer results}

Two examples of spin configurations in zero field are presented. Fig. 1 shows the relaxed spin configuration of a stripe domain, in which initially one half section had spin up, the other half spin down and of a random start configuration.

Fig. 2 shows the hysteresis loops obtained with both iteration methods.

The coercive fields are approximately $200 \mathrm{kAm}^{-1}$ The remanent magnetisation are approximately $0.8 \mathrm{~m}$, and $0.9 m_{\mathrm{s}}$ for, respectively, the methods using energy minimalisation and the equation of motion. For both methods the results are roughly the same, although the method using the LLG equations needs many more integrations (20000, compared with 5000 (CPU-times resp. $10.2 \mathrm{~h}$ )) for a convergent hysteresis loop.

\section{Conclusions}

For hysteresis loops the simulations using LLG equations or energy minimalisation are roughly the same, although the energy minimalisation method is favourable because less integrations are needed. The influence of the damping parameter $\alpha$ in the LLG-equations and the other parameters on spin configurations and hyster- 
esis loops will be studied further in detail. Future research will concern simulations of multi-layer structures, like $\mathrm{CoCr} / \mathrm{NiFe}$ double layers and $\mathrm{CoCr}$ films with an initial and bulk layer. Calculations of the absorption of radiation by ferromagnetic layer, using an harmonic component added to the external field, are planned.

\section{References}

[1] A.H. Morrish, The Physical Principles of Magnetism (John Wiley, New York, 1966) p. 284.

[2] M.E. Schabes and H.N. Bertram, J. Appl. Phys. 64 (1988) 1347.

[3] J.-G. Zhu and H.N. Bertram, J. Appl. Phys. 63 (1988) 3248. 